\title{
Myelodysplastic syndromes
}

\author{
Ian M Hann
}

Myelodysplasia is one of the Cinderella subjects of paediatric haematology and oncology. Although cases are rare and probably do not account for more than $3 \%$ of haematological malignancies, patients presenting with these disorders pose a very serious challenge to successful diagnosis and management with which we are only just coming to grips. In fact, such is the previous state of confusion that we have no idea of the prevalence of the component disorders and there is little doubt that under diagnosis was itself prevalent. The first tentative steps have now been taken out of the darkness and probable cures are being achieved in previously almost uniformly fatal conditions. The first moves in the right direction were achieved by abandoning the paediatricians inherent love of syndromes and concentrating, with our adult haematology colleagues, on getting some sense and science into classification of the disorders.

\section{Classification}

The basic concept of myelodysplasia is a very simple one: the bone marrow is active but ineffective. Thus, there are functional morphological abnormalities and/or pancytopenia. The syndromes thus include the disorders previously called refractory anaemia, smouldering leukaemia, and preleukaemia all of which had a strong predilection to progress to acute myeloid leukaemia at varying pace. One series of childhood acute myeloid leukaemia has suggested that $17 \%$ of cases had a preleukaemic phase. ${ }^{1}$

The French-American-British cooperative morphology group has led the way in providing the systematic groundwork for a classification system. ${ }^{2}$ Subsequently the Morphologic, Immunologic and Cytogenetic Cooperative Study Group has provided additional cytogenetic information which has allowed further refinement. ${ }^{3}$ The conventional approach has been to distinguish myeloproliferative disorders (such as chronic granulocytic leukaemia, essential thrombocythaemia, myelofibrosis, and polycythaemia) from myelodysplasia. This is a somewhat artificial distinction, bearing in mind that all are clonal proliferations and all may evolve to a frank acute leukaemia. However, it forms the basis for the best attempts at classification currently available for childhood myelodysplasia ${ }^{4}$ (see table 1) and eventually a better classification will hopefully come out in the biological wash. This includes the two commonest syndromes described in the paedia- tric literature, juvenile chronic myeloid leukaemia (JCML) and infantile monosomy 7 syndrome. Both of these disorders will presumably take their place under more appropriate headings when more systematic studies are completed, for example, JCML shows some features akin to a well described adult counterpart, chronic myelomonocytic leukaemia (CMML). Table 2 shows a recommended list of investigations required for the diagnosis and classification of myelodysplasia.

I would like to expend a little space on the specific features of the disorders listed in table 1 . However, there is insufficient space to deal adequately with chronic myeloid leukaemia, which has been extensively reviewed elsewhere. ${ }^{5-10}$ Essential thrombocythaemia and polycythaemia are excessively rare in childhood and the few authenticated cases have also been reviewed elsewhere. ${ }^{11-13}$ Malignant myelofibrosis as seen in adults ${ }^{14} 15$ is not seen in children and modern techniques have shown

\section{Table 1 Classification of paediatric myelodysplasia}

\begin{tabular}{l} 
- Primary myelodysplasia: \\
Juvenile chronic myeloid leukaemia \\
Infantile monosomy 7 syndrome \\
Refractory anaemia \\
Refractory anaemia with excess of blasts \\
Refractory anaemia with excess of blasts in transformation \\
- Proliferative myelodysplasia: \\
Down's syndrome leukaemoid reactions \\
Myelodysplasia with eosinophilia \\
Philadelphia positive chronic myeloid leukaemia \\
Atypical chronic myeloid leukaemia \\
Familial myelodysplasia \\
Essential thrombocythaemia \\
Polycythaemia rubra vera \\
- Secondary myelodysplasia: \\
Familial \\
Therapy induced \\
\hline Adapted from Chessells.
\end{tabular}

Table 2 Investigation of myelodysplasia

- Blood:

Haemoglobin + red cell indices

White cell count + differential

Blood film

Fetal haemoglobin concentration

Immunoglobulins

- Bone marrow:

Aspirate + trephine biopsy

Cytochemistry and iron stain

Cytogenetics

- Additional tests:

Neutrophil function

Platelet function

Lymphocyte subsets

Tissue culture marrow colony assays

Antinuclear antibodies

Fetal globin glycine:alanine ratio

Carbonic anhydrase

Adapated from Chessells. 
that primary myelofibrosis is in fact acute megakaryoblastic leukaemia wherein the proliferating megakaryocytes induce extensive marrow fibrosis. ${ }^{16}$

Juvenile chronic myeloid leukaemia

JCML does appear to be a distinct disease entity, the pathognomonic feature of which is a reversion to a truly fetal haemopoiesis with raised concentrations of fetal haemoglobin above $10 \%,{ }^{17}$ a fetal pattern of red cell enzymes, ${ }^{18}$ and in one reported case the persistence of epsilon globin chains that normally disappear by 3 months of age. ${ }^{19}$ The disorder appears to be more common in boys, and children with neurofibromatosis show a higher number of cases than expected of JCML and chronic myeloid leukaemia. ${ }^{20} 21$ The usual clinical presentation is with pallor, splenomegaly, and bleeding due to thrombocytopenia. The latter (along with the higher fetal haemoglobin concentration) distinguishes this disorder from monosomy 7 myelodysplasia (see below). ${ }^{17}$ Cutaneous xanthomata occasionally occur ${ }^{20}$ and led to the outdated description of 'xantholeukaemia'. There is often a facial rash in a butterfly distribution that may also involve the trunk, predate the other findings, and lead to a misdiagnosis of lupus erythematosus, especially as antinuclear antibodies may be present. ${ }^{22}$

Since the original reports of this disorder ${ }^{23}$ it has become standard practice to compare and contrast this disorder with Philadelphia chromosome positive chronic myeloid leukaemia. This is a largely pointless exercise and in fact the morphological and some other features, for example the immune disturbance, ${ }^{22}$ are more akin to CMML as previously described in adults. Further evidence is provided by the presence of monocyte specific antigens on the surface of mononuclear cells ${ }^{23}$ and outgrowth of macrophage colonies in tissue culture. ${ }^{24}$ However, the bone marrow may show various cytogenetic abnormalities or be normal and thus there are no diagnostic cytogenetic findings. ${ }^{25}$ This is in contrast to monosomy 7 myelodysplasia which can otherwise be indistinguishable, ${ }^{26}$ although JCML is more frequently associated with a lower platelet count as well as the raised fetal haemoglobin. ${ }^{27}$ As with many of the myelodysplastic disorders in childhood, more can be gleaned from an examination of the blood film than the bone marrow aspirate. The white cell count is usually only moderately raised and rarely above $50 \times 10^{9} / 1$, anaemia is common, the platelet count reduced, and there is usually a prominent monocytosis often associated with dysplastic granulocytes and some blast cells. The bone marrow findings are similar but usually much less noticeable. The prognosis is very poor and over the last 20 years we have seen 14 children at Great Ormond Street, 11 boys and three girls with a median age of 3 years and median survival of only six months. These patients did not develop a terminal leukaemic 'blast crisis' as is sometimes described, but developed progressive weight loss, nodal enlargement, and some increase in blasts and normoblasts.
Monosomy 7 myelodysplasia

Monosomy and partial deletion of chromosome 7 is found in primary myelodysplasia as described here, but also in de novo acute myeloid leukaemia, secondary myelodysplasia, and secondary acute myeloid leukaemia. In each of these disorders there is a defect in neutrophil chemotaxis and killing leading to a particular susceptibility to bacterial infection. ${ }^{28}$ The presence of the deletion in biphenotypic leukaemias adds weight to the proposition that monosomy 7 is associated with transformation of a common lymphoid-myeloid progenitor cell. ${ }^{29}$ The variability of clinical features in association with abnormalities of chromosome 7 are well illustrated by the experience at this hospital over the last 20 years. During this period we have seen four cases of de novo acute myeloid leukaemia, one of secondary acute myeloid leukaemia, one of myelofibrosis with myelodysplasia, one of refractory anaemia with excess of blasts with transformation (RAEB-t), and 13 children with the myeloproliferative syndrome. It is important to note that there are families with an apparent predilection to the development of acute myeloid leukaemia or myelodysplasia and this may be associated with chromosome 7 abnormalities. ${ }^{30}$ The 13 children we have looked after with monosomy 7 myelodysplasia presented at a median age of 10 months but the oldest was aged 8 years and had an indolent process of recurrent infections; hypogammaglobulinaemia, and monocytosis over a six year period. Three of the 13 developed acute myeloid leukaemia and one developed massive splenomegaly and marrow fibrosis. The time to the development of acute myeloid leukaemia varied from three months to two years. A third of the cases survived more than two years and thus this disorder is associated with longer survival than JCML with a predilection to the development of acute myeloid leukaemia.

\section{Other myelodysplasias: primary}

The current lack of knowledge does not allow a calculation of the prevalence of these disorders. Several series have highlighted the paucity of children who present with the types of myelodysplasia associated with a better prognosis, that is refractory anaemia (RA) and refractory anaemia with ringed sideroblasts (RARS). ${ }^{31} 32$ We have seen only two cases of RARS, both of which followed a chronic course. Thus, the vast majority of children with myelodysplasia unfortunately fall into the bad risk categories of RAEB with or without transformation towards acute myeloid leukaemia. All series consistently show a less than $20 \%$ chance of survival to five years and there are very few long term survivors. However, there are encouraging reports demonstrating a survival of up to $50 \%$ after bone marrow transplantation for those who have a histocompatible sibling. ${ }^{33}$ Attempts have been made to identify features associated with prognosis in adults. Features predictive of long survival include pancytopenia, more than $5 \%$ blasts in the blood, abnormal localisation of immature precursors on a trephine biopsy 
specimen, and a karyotype showing complex chromosomal abnormalities. ${ }^{34}$

It has been known for many years that children with Down's syndrome are at risk of a neonatal myeloproliferative syndrome resembling acute leukaemia, which usually undergoes spontaneous remission. Some of these children later develop acute leukaemia after an interval ranging from six months to three years. ${ }^{35}$ In addition, children with Down's syndrome and acute myeloid leukaemia can have a prodromal myelodysplastic phase lasting several months with occasional blasts seen on the blood film. There is an obvious need to follow up a series of babies with Down's syndrome in order to determine the true incidence of this transient abnormal myelopoiesis and the risk of development of acute leukaemia. Clinically the disorder resembles congenital leukaemia with hepatosplenomegaly but without the skin infiltration seen in the former. ${ }^{36}$ There is a blood leukocytosis with many blast cells, which have recently been shown to be megakaryoblasts. ${ }^{37}$ Although these children may develop hyperviscosity problems due to the high white blood cell count, they do not develop severe bone marrow failure although they may need some red cell and platelet transfusions. The management is conservative with supportive care only and the most important thing is to follow up the patients carefully in case they develop acute myeloid leukaemia.

A number of children have presented with hepatosplenomegaly, dramatic eosinophilia, myelodysplasia, and cytogenetic abnormality involving the area of chromosome $5 q 31$ that contains the gene for interleukin-5 (IL-5 'eosinopoietin'). ${ }^{38}$ We have a patient in whom there is a prolonged history of a severe infiltrative skin disorder and dramatic eosinophilia.

There is a 'ragbag' of other very rare myeloproliferative disorders in childhood. Several have been shown to involve translocations of chromosome $11 \mathrm{p} 15 .{ }^{39}$ Rarely, cases otherwise identical to chronic myeloid leukaemia but without the Philadelphia chromosome and with no bcr-abl gene rearrangement ${ }^{34}$ are seen in childhood. A morphological review of childhood cases is required and it is possible that they will be classified with CMML. The prognosis of this disorder is said to be poor.

\section{Other myelodysplasias: secondary}

Children with congenital bone marrow disorders such as Fanconi's anaemia and Shwachman's syndrome can present with or develop myelodysplasia as well as acute myeloid leukaemia. ${ }^{40} 41$ Unfortunately we are also seeing an increased incidence of acute myeloid leukaemia and myelodysplasia in patients treated for malignant disease, most commonly Hodgkin's disease but also non-Hodgkin's lymphoma and other solid tumours. The alkylating agents are most clearly implicated in this susceptibility. ${ }^{42}$ The risk of secondary leukaemia and myelodysplasia after treatment for acute lymphoblastic leukaemia is not clear but so far there have only been a few cases reported in the UK.

Cytogenetic abnormalities are found in almost all children with secondary myelodysplasia/acute myeloid leukaemia and are frequently multiple, the majority involving chromosomes 5 and 7 with 3 and 17 being less frequently involved. The chromosome 5 abnormalities always involve 5q23-32, a region containing the genes for several growth factors and their receptors. ${ }^{43}$

Clearly the most important point to be made here is that the planning of treatment for malignant disease should take into account the need to try to avoid the use of alkylating agents where alternative treatments are possible and especially to try to avoid their concomitant use with radiotherapy. This has proved possible with regimens for low grade non-Hodgkin's lymphoma. The published results of treatment for secondary myelodysplasia make depressing reading. Patients with chromosome 5 abnormalities did particularly badly in the past. Remission duration, if achieved at all, tends to be extremely short in this whole group of children before the era of bone marrow transplantation. ${ }^{42}$

\section{Biology of myelodysplasia}

Various modern techniques have demonstrated that the myelodysplasias are clonal disorders arising in a multipotent or pluripotent stem cell, ${ }^{44}$ and the blood cells produced often survive for a short time and have abnormal function. Disease progression is a function of the expansion of the abnormal clone with progressively abnormal blood count and/or development of acute myeloid leukaemia. This process is frequently associated with immunological abnormalities including raised immunoglobulins, reduced numbers of natural killer cells, and an abnormal helper:suppressor $\mathrm{T}$ cell ratio. ${ }^{45} \mathrm{~A}$ variety of abnormalities of bone marrow growth in colony cultures has been described. In children one of the commonest phenomena is spontaneous growth of colony forming unit-granulocyte/macrophage and other patients show reduced growth of multipotent progenitor cells. ${ }^{26}$

A large number of attempts have been made to identify factors associated with disease progression towards acute myeloid leukaemia. One promising line of inquiry relates to the ras group of genes which are involved in the regulation of cell proliferation. Preliminary studies have in fact shown that these genes are activated in patients with myelodysplasia and possibly more so in those who develop acute myeloid leukaemia. ${ }^{46}$ The other main line of scientific investigation involves the possible role of putative repressor genes in the regions of chromosomes 5 and 7 frequently involved in myelodysplasia. The hypothesis is that loss of the normal allele could result in the unmasking of a recessive mutant allele on the remaining chromosome, as occurs in retinoblastoma. The relevant chromosomal regions certainly contain the genes for a tantalising array of growth factors and other important compounds-for example, 5q23-31 contains granulocyte/macrophage-colony stimulating factor (GM-CSF), interleukin 3, macrophage-colony stimulating 
factor (M-CSF), and $c$-fms (the M-CSF receptor). Similarly $7 q$ is the site of the erythropoietin gene, the met proto-oncogene, and the multidrug resistance gene (MDR-1). ${ }^{46}$

\section{Treatment options}

Having completed the investigations detailed in table 2 , one is then faced with several dilemmas. Most children with myeloproliferative/myelodysplasia have an extremely poor prognosis although occasionally there is a long prodromal phase during which the patient can be very well; Philadelphia chromosome positive chronic myeloid leukaemia is the best example of this phenomenon. It is in fact quite easy sometimes to be lulled into a false sense of security when in fact a knowledge of the extremely poor prognosis should stimulate a search for possible curative options. The management of chronic myeloid leukaemia has been very well reviewed elsewhere ${ }^{5-10}$ and basically comes down to looking for one type of bone marrow transplantation or another. Looking after these children is a great challenge for paediatric haematologists and I will briefly review the limited therapeutic armamentarium.

Various chemotherapeutic regimens have been tried in myelodysplasia with palliative and curative intent. Other than using intensive chemotherapy for patients who effectively have acute myeloid leukaemia these have been singularly unsuccessful in achieving the latter goal. ${ }^{47}$ However, we have now given six children with infantile monosomy 7 myelodysplasia combination chemotherapy and two remain in remission four and five years from chemotherapy. ${ }^{48}$ The lesson to be learnt here, applicable in other myelodysplasias, is that early treatment can be successful but waiting until the development of acute myeloid leukaemia appears to reduce the likelihood of a good outcome. A reasonable palliative effect can be achieved with combinations such as cytarabine and mercaptopurine ${ }^{49}$ with some reduction of bulk disease and requirements for blood product support. These patients usually require intensive supportive care with antimicrobial agents, blood, and platelets especially if the plan is to attempt a curative bone marrow transplantation

There is a current vogue for cytokine treatment and many consequent publications. ${ }^{50}$ The most commonly used at present are GM-CSF and granulocyte-colony stimulating factor. To cut a long story short, it is usually possible to improve neutropenia but not thrombocytopenia. The worry of course is that the neutrophil dysfunction will not be improved and the underlying disease will be 'cranked up'. Randomised trials are in progress so we should eventually know if these expensive compounds will help. It is also interesting to note that patients with JCML have abnormal cytokine production and regulation, which opens up the possibility of treatment with inhibitors or regulators of cytokines when these become available.

Bone marrow transplantation

As previously stated, myelodysplasia with a good prognosis (basically refractory anaemia and RARS) is a rarity and the main consideration is, having improved the child's general health as far as possible, whether a donor for bone marrow transplantation is available and if not whether a matched unrelated donor can be found on the various donor registries. The decision to go ahead with bone marrow transplantation using a matched unrelated donor can be difficult especially in a currently well child because of the relatively poor results and high mortality at present. However, techniques are improving and for almost all patients it will be their only chance of cure.

There are few published reports of the outcome of bone marrow transplantation for myelodysplasia and even fewer related to children. Matched allogeneic transplants have been associated with survival beyond three years of approximately $45 \%$. The evidence is limited but bone marrow transplantation is undoubtedly the most effective treatment for myelodysplasia in adults and children. What is not known yet is whether one can get away with a chemotherapy 'conditioning' regimen rather than the conventional cyclophosphamide plus total body irradiation approach which is associated with major endocrine, growth, and other late effects. Preliminary studies in childhood chronic myeloid leukaemia suggest that the chemotherapy only approach may be suitable especially for very young patients. ${ }^{51}$ Successes in bone marrow transplantation for JCML have been reported from Seattle with three of six children receiving a graft from a compatible sibling and three of eight using a donor with a 1-3 antigen mismatch alive and in remission after the use of cyclophosphamide and total body irradiation. ${ }^{52}$ In this situation one case report suggests that a chemotherapy only regimen may not eradicate the malignant clone. ${ }^{53}$ We have also performed a successful bone marrow transplantation in a child with monosomy 7 myelodysplasia using a histocompatible sibling and a chemotherapy/total body irradiation regimen.

The problem is that only one in three children will have a histocompatible sibling donor and now great efforts are being made to improve the chances of finding a matched unrelated donor rapidly and carrying out a successful bone marrow transplantation. Results in children with chronic myeloid leukaemia show an acturial event free survival of $30 \%$ at 30 months,${ }^{54}$ suggesting that this approach should be considered for all patients who do not have a sibling donor.

\section{Conclusion}

I hope that I have made it clear that the myelodysplastic syndromes are worthy of more attention both scientifically and clinically. We badly need more studies designed to determine the real prognosis and incidence of these disorders and to test the efficacy of modern treatments such as bone marrow transplantation and cytokine therapy. At a more fundamental level scientific investigations should lead to a better basic understanding of leukaemogenesis. 
At long last we are beginning to understand more and to cure some of the patients but for the foreseeable future any child presenting with myelodysplasia will continue to tax our understanding and management skills to the limit.

Many thanks are due to Professor Judith Chessells, withou whose help and guidance this article could not have been produced.

1 Blank J, Lange B. Preleukemia in children. $f$ Pediat

2 Bennett JM, Catovsky D, Daniel MT, et al. Proposals for the classification of the myelodysplastic syndromes. $\mathrm{Br} \mathcal{J}$ Haematol 1982;51:189-99.

3 Third MIC Co-operative Study Group. Recommendations fo a morphologic, immunologic and cytogenetic (MIC) working classification of the primary and therapy-related myelodysplastic disorders. Cancer Genet and Cytogenet 1988;32:1-10.

4 Chessells JM. Myelodysplasia. In: Hann IM, Gibson BES, eds. Clinical haematology paediatric haematology. Vol 4 No 2: 459-482. London: Baillière-Tindall, 1991 .

5 Goldman JM, ed. Chronic myeloid leukaemia. London: Baillière-Tindall, 1987

6 Klingebiel T, Creutzig U, Dopfer R, et al. Bone marrow transplantation in comparison with conventional therapy in children with adult type chronic myelogenous leukemia. children with adult type chronic myeloge
Bone Marrow Transplant 1990;5:317-20.

7 Offit K, Burns JP, Cunningham I, et al. Cytogenic analysis of chimerism and leukemia relapse in chronic myelogenous leukemia patients after $T$ cell-depleted bone marrow transplantation. Blood 1990;75:1346-55.

8 Hirsch-Ginsberg C, LeMaistre AC, Kantarjian H, et al. RAS mutations are rare events in Philadelphia chromosomenegative/bcr gene rearrangement-negative chronic myelogenous leukemia, but are prevalent in chronic myelomonocytic leukemia. Blood 1990;76:1214-9.

9 Tien HF, Wang CH, Chen YC, et al. Chromosome and bcr rearrangement in chronic myelogenous leukaemia and their correlation with clinical states and prognosis of the disease. Br f Haematol 1990;75:469-75.

10 Nelkin BD, Przepiorka D, Burke PJ, et al. Abnormal methylation of the calcitonin gene masks progression of methylation of the calcitonin gene masks progression
chronic myeloid leukaemia. Blood 1991;77:2431 -4 .

11 Fernandez-Robles E, Vermylen C, Martiat P, et al. Familia essential thrombocythemia. Pediatr Hematol Oncol 1990;7: 373-6.

12 Chintagumpala MM, Steuber CP, Mahoney DH, et al. Essential thrombocythemia in a child: management with anagrelide. Am f Pediatr Hematol Oncol 1991;13:52-6.

13 Schwartz CL, Cohen HJ. Preleukaemic syndromes and other syndromes predisposing to leukaemia. Pediatr Clin North Am 1988;35:853-71.

14 Donti E, Tabilio A, Bocchini F, et al. Partial trisomy lq in idiopathic myelofibrosis. Leuk Res 1990;14:1035-40.

15 Lambertenghi-Deliliers G, Orazi A, Luksch R, et al. Myelodysplastic syndrome with increased marrow fibrosis: a distinct clinico-pathological entity. Br f Haematol 1991;78 161-6.

16 Bennett JM, Catovsky D, Daniel M-T, et al. Criteria for the diagnosis of acute megakaryocytic leukaemia (M7). Ann Intern Med 1985;103:460-2.

17 Weatherall DJ, Edwards JA, Donohoe WTA. Haemoglobin and red cell enzyme changes in juvenile chronic myeloid and red cell enzyme changes in juve

18 Travis SF. Fetal erythropoiesis in juvenile chronic myelocytic leukaemia. Blood 1983;62:602-5.

19 Weinberg RS, Leibowitz D, Weinblatt ME, et al. Juvenile chronic myelogenous leukaemia: the only example of truly fetal (not fetal-like) erythropoiesis. Br f Haematol 1990;76: 307-10.

20 Merys JA, Beerhout RC, Bagby GC, Koler RD. Juvenile chronic granulocytic leukaemia. Am $\mathcal{F}$ Dis Child 1980;134: 654-8.

21 Bader JL, Miller RW. Neurofibromatosis and childhood leukaemia. 7 Pediatr 1978;92:925-9.

22 Cannat A, Seligmann M. Immunological abnormalities in juvenile myelomonocytic leukaemia. BMF 1973;i:171-4.

23 Hardisty RM, Speed DE, Till M. Granulocytic leukaemia in childhood. Br $\mathcal{F}$ Haematol 1964;76:1-6.

24 Gualtieri RJ, Emanuel PD, Zuckerman KS. Granulocytemacrophage colony stimulating factor is an endogenous regulator of cell proliferation in JCML. Blood 1989;74: regulator

25 Kaneko Y, Maseki N, Sakurai M, et al. Chromosome pattern in juvenile chronic myelogenous leukaemia, myelodysplastic syndrome and acute leukaemia associated with neurofibromatosis. Leukemia 1989;3:36-41.

26 Brandwein JM, Horsman DE, Eaves AC, et al. Childhood myelodysplasia: suggested classification as myelodysplastic syndromes based on laboratory and clinical findings. $A m \mathcal{F}$ Pediatr Hematol Oncol 1990;12:63-70.

27 Sieff CA, Chessells JM, Harvey BAM, et al. Monosomy 7 in childhood: a myeloproliferative disorder. $\mathrm{Br} \mathcal{F}$ Haemato 1981;49:235-49.

28 Ruutu P, Ruutu T, Vuopi I, et al. Defective chemotaxis in monosomy 7. Nature 1977;265:146-7.

29 Chan LC, Sheer D, Drysdale HC, et al. Monosomy 7 and multipotential stem cell transformation. $B r f$ Haematol 1985;61:531-9.

30 Carroll WL, Morgan R, Glader BE. Childhood bone marrow monosomy 7 syndrome: a familial disorder? $\mathcal{f}$ Pediatr 1985;107:578-80.

31 Wegelius R. Preleukaemic states in children. Scandinavian Fournal of Haematology 1986;36:133-9.

32 Creutzig U, Cantu-Rajnoldi A, Ritter J, et al. Myelodysplastic syndromes in childhood. Report of 21 patients from Italy and West Germany. Am $\mathcal{F}$ Pediatr Hematol Oncol 1990;9: 324-30.

33 Guinan EC, Tantravali R, Weinstein HJ. Bone marrow transplantation for children with myelodysplastic syndromes. Blood 1989;73:619-22.

34 Mufti GJ, Galton DAG. Myelodysplastic syndromes: natura history and features of prognostic importance. Clinics in Haematology 1986;15:953-71.

35 Bain B. Down's syndrome-transient abnormal myelopoiesis and acute leukaemia. Leukemia and Lymphoma 1991;3: and acu

36 Barnett PLJ, Clark ACL, Garson OM. Acute non-lymphocytic leukemia after transient myeloproliferative disorder in a patient with Down's syndrome. Med Pediatr Oncol 1990 18:347-53.

37 Eguchi $M$, Sakakibara H, Suda J, et al. Ultrastructural and ultracytochemical differences between TAM and megakaryoblastic leukaemia in Down's syndrome. $\mathrm{Br}$ f Haemato 1989;73:315-22.

38 Darbyshire PJ, Shortland DB, Swansbury JG, et al. A myeloproliferative disorder in two infants associated with eosinophilia and chromosome $\mathrm{t}(1 ; 5)$ translocation. $\mathrm{Br} f$ Haematol 1987;6:483-6.

39 Inaba T, Hayashi Y, Hanada R, et al. Childhood myelodysplastic syndromes with $11 \mathrm{p} 15$ translocation. Cancer Genet Cytogenet 1988;34:41-6.

40 Auerbach AD, Weiner MA, Warburton D. Acute myeloid leukaemia as the first haematologic manifestation of Fanconi anaemia. Am $\mathcal{f}$ Hematol 1982;12:289.

41 Woods WG, Roloff JS, Lukens JN, Krivit W. The occurrence of leukaemia in patients with Shwachman syndrome. of leukaemia in patient

42 Levine EG, Bloomfield CD. Secondary myelodysplastic syndromes and leukaemias. Clinics in Haematology 1986;15 1037-80

43 Le Beau MM, Albain KS, Larson RA, et al. Clinical and cytogenetic correlations in 63 patients with therapy-related MDS and AML. F Clin Oncol 1986;4:325-45.

44 Tefferi A, Thibodeau SN, Solberg LA. Clonal studies in the myelodysplastic syndromes using X-linked restriction fragment length polymorphisms. Blood 1990;75:1770-3.

45 List AF, Ganewal HS, Sandberg AA. The myelodysplastic syndromes: biology and implications for management. syndromes: biology ancol $1990 ; 8: 1424-41$.
Clin

46 Jacobs A. Oncogenes in the myelodysplastic syndrome. Blood Rev 1989;3:105-9.

47 De Witte T, Muus P, De Pauw B, Hannen C. Intensive antileukaemic treatment of patients younger than 65 years antileukaemic treatment of patients younger than 65 years

48 Evans JPM, Czepulkowski B, Gibbons B, et al. Childhood monosomy 7 revisited. Br 7 Haematol 1988;69:41-5.

49 Lilleyman JS, Harrison JF, Black JA. Treatment of juvenile chronic myeloid leukaemia with sequential subcutaneous cytarabine and oral mercaptopurine. Blood 1977;49: 559-62.

50 Cheson BD. The myelodysplastic syndromes: curren approaches to therapy Ann Intern Med 1990;112:932-41.

51 Tutschka PJ, Copelan EA, K plantation for leukaemia following a new busulfan and cyclophosphamide regimen. Blood 1987;70:1382-8.

52 Sanders JE, Buckner CD, Thomas ED, et al. Allogeneic marrow transplantation for children with juvenile chronic myeloid leukaemia. Blood 1988;71:1144-6.

53 Urban C Schwinger W, Slave I, et al Busulfan/cyclophosphamide plus BMT is not sufficient to eradicate the phalignant clone in juvenile Bone Marrow Transplant 1990;5:353-6.

54 McGlave PB, Beatty P, Ash R, Hows JM. Therapy fo chronic myelogenous leukaemia with unrelated donor bone marrow transplantation: results in 102 cases. Blood 1990; 75:1728-32. 